\section{Technology-Driven Physics Action}

Following the recommendations of an ad hoc working group, four areas have been proposed for a new COST Action in Technology -Driven Physics. A technical committee is now seeking detailed descriptions of the activities that should be considered for inclusion. Groups from industry as well as from basic research institutes are encouraged to make proposals since industry will benefit from an improved understanding of complex processes, materials and devices while research institutions can profit from improved collaboration.

The main topics covered by each of the proposed Action's four areas are summarised below, together with some suggested subjects. If you are interested in establishing the new Action, or in making a proposal for an activity that should be included, please contact the appropriate technical coordinator.

\section{- Modelling of Physical Phenomena in Technological Applications}

Prof. M. Deville, Institut de Machines Hydrauliques et de Méchanique des Fluides, EPFL, CH-1015 Lausanne (tel: +41-21-693 53 18; fax: +41-21-6933646; deville@dgm.epfl.ch)

Applying computational science to solving problems in science and technology is proving an effective way to generate new knowledge. Approaches which can be applied technologically must be developed in areas covering the entire spectrum of modelling that involves condensed-matter and non-linear physics as well as materials science.

- Physics, mathematical modelling, simulation, algorithms, advanced computer architectures, software engineering tools, networking, visualisation of databases. - Basic physics of condensed matter: e.g., Monte Carlo techniques.

- First-principles atomistic simulations.

- Micromechanical simulations: crack tips, dislocations, etc.

- Ferromagnetic materials: magnets, recording media, etc.

- Multicomponent systems: prediction of macroscopic behaviour.

\section{COST Actions}

European Cooperation (concerted action) in Scientific and Technical Research (COST) is a framework for coordinated national research at the European level among the 25 COST Member States set up in 1971. Public-and private-sector organizations in non-COST countries have been able to participate since 1981 if there is a justified mutual interest.

Flexible à la carte COST Actions consist of basic and precompetitive research as well as activities of public utility that are funded by the Member States. European Union (EU) institutions play an important role, but in contrast to EU programmes, COST collaboration does not require an agreed overall policy: it focuses instead on specific themes for which there is a particular interest, usually expressed via a bottom-up approach. So COST is complementary to EUREKA and the EU's Framework, with the EU paying coordination costs.

Today there are 115 COST Actions in 15 disciplines, each involving at least five Member States, managed by a Management Committee and lasting on average five years. The total national funding for COST Actions averages about 5000 MECU p.a., and acceptance in a COST

\section{- Applications of Non-linear Optical Phenomena}

Prof. Y. Lion, Lab. de Physique Générale, Institut de Physique, , Bât. B5, Université de Liège, B-4000 SartTilman (tel.: +32-41-66 36 26; fax: +32-41-66 23 55; lion@gw.unipc.ulg.ac.be)

Detailed studies of the physics underlying non-linear optical phenomena are needed. - Photoactive elements and devices: optical neural networks, optical storage systems, optical switches based on, e.g., photochromic dyes; spectral hole burning. - Integrated optics: systems with artificial non-linearities, e.g., periodically poled fibres, quasi-phase matched crystals; multipolar structures, e.g., new semiconductor micro- and nano-structures; new waveguides, e.g., hybrid structures.

- Laser technology: New lasers based on nonlinearity; scaling of semiconductor diode lasers to multi-watt powers; bluegreen semiconductor lasers; microcavities; new organic materials, e.g., glass fibres, rare-earth ions in crystals.

- Characterization of optical materials: development to industrial standards of techniques for non-linear optical materials, e.g., z-scan, four-wave mixing, etc.
COST: further details

M. Chapuis, COST Secretariat European Commission, Brussels Tel: $+32-2-2957147$ Fax: +32-2-2964289

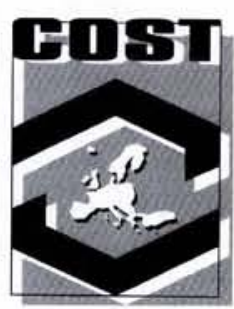

Action often facilitates national funding of a project.

Only the Application of lon-beam Analysis Action involves physics directly. Following the recommendation of an ad hoc working group, COST's main authority - the Committee of Senior Officials (CSO) - has appointed a technical committee to help start up a proposed Technology-Driven Physics Action. The committee would normally become a New Action Group of interested national coordinators and technical experts charged with establishing the basis of the proposed Action, by having the technical annex of a Memorandum of Understanding $(\mathrm{MoU})$ approved by National Coordinators and eventually by the CSO. The Action gets underway once five Member States sign the MoU.

\section{- Non-linear Dynamics of Mechanical Processing}

Prof. I. Grabec, Faculty of Mechanical Engineering, Univ. of Ljubljana, POB 394, SI-1000 Ljubljana (tel: +386-61-177 12 00; fax: +386-61-21 85 67; igor.grabec@fs.uni-lj.si)

Advances in the physics of complex nonlinear systems need to be combined with the experimental study, modelling, control, and optimization of mechanical processes. Research should focus on the instabilities and non-linear features which arise in materials-processing systems, with an emphasis on chaotic behaviour. The aim is to explain the origin of dynamic self-reorganization of unstable flows in processed materials and the formation of surfaces, patterns and structures.

- Physics of mechanical shaping processes: cutting, plastic forming, forging; processing in sheet, powder and semi-solid forms; casting, pressing and extruding.

- Interaction between manufacturing machinery and workpieces, especially chaotic behaviour.

- Materials under non-equilibrium conditions: combined modelling of fluid and plastic flows.

- Adaptive modelling and control of nonlinear and chaotic mechanical processes: structural and surface properties of workpieces; interaction between the workpiece and tools, the operating system and the machine structure.

\section{Soft Condensed Matter}

Dr. T. Michels, Shell Res. \& Technology Centre, POB 38000, NL-1030 BN Amsterdam (tel: + 31-20-630 40 41; fax: +31-20-63020 47; michels1@siop.shell.nl)

Soft condensed matter covers a wide class of materials present in daily life as industrial products and natural substances. Further understanding needs physics research of a generic nature that is focussed by selecting a few fundamental subjects. - Physics of bipolymers and food: macromolecular conformation versus function; association and kinetics of structural change; thermodynamics and kinetics of structural change.

- Polymer ordering and micromechanics: kinetics and morphology of polymer crystallization; mesoscale structure versus mechanical strength; microstructural and mechanical degradation.

- Rheology of structured fluids: aggregating and gel-forming suspensions; dense powders and pastes; foams.

- Characterization of SCM structures: small-angle scattering; atomic-force microscopy of polymer surfaces; optical and electromagnetic resonance. 